\title{
DISPOSITIVOS DE CUIDADO COM A SAÚDE PSÍQUICA DE PROFISSIONAIS DA ATENÇÃO PRIMÁRIA NO BRASIL
}

\author{
Mental health care devices of Primary Health professionals in Brazil
}

Dispositifs de soins avec la santé mentale des professionnels de soins primaire au Brésil

Dispositivos de cuidado de la salud mental de profesionales de la Atención Primaria en Brasil

Luciana Oliveira dos Santos $^{1}$

Professora do Departamento de Saúde Coletiva da UnB, possui Doutorado e Mestrado em Saúde Coletiva pelo Instituto de Medicina Social da Universidade do Estado do Rio de Janeiro e graduação em Psicologia pela

Universidade Federal de Pernambuco. Pesquisadora as sociada ao Laboratório de Psicodinâmica e Clínica do Trabalho do Instituto de Psicologia da UnB, Professora titular da UNIP (Universidade Paulista) - Campus Brasília e do CBPEX (Consultoria Brasileira de Ensino, Pesquisa e Extensão) - Brasília.

Loyanne Chaves Ferreira da Silva ${ }^{2}$

Graduada em Saúde Coletiva pela Universidade de Brasília em 2017, com desenvolvimento de estudos e pesquisas na área do trabalho em saúde e gerenciamento de unidades de saúde da atenção primária em saúde no

Magda Duarte dos Anjos Scherer ${ }^{3}$

Pós doutorado no Conservatoire des Arts et Metiers (CNAM) (Bolsista CAPES 2013/2014) ; Doutorado em Enfermagem pela Universidade Federal de Santa Catarina (2006); especialização e mestrado em Saúde Pública pela Fundação Oswaldo Cruz; e graduação em Serviço Social pela Universidade Federal de Santa Catarina (1983). Líder do Grupo de Estudos e Pesquis as sobre o Trabalho em Saúde, CNPq, Brasil.

\section{RESUMO}

O trabalho em saúde se caracteriza por ser um campo repleto de conflitos subjetivos, ocasionando situações de sofrimento nos profissionais, que podem resultar em adoecimento psíquico, como é o caso da atenção primária, onde o objeto do trabalho é de grande complexidade, exigindo lidar com situações que muitas vezes fogem da capacidade e da competência do profissional. Também influencia no adoecimento do trabalhador o modelo organizacional adotado. Sendo assim, desenvolveu-se uma revisão integrativa visando mapear dispositivos de cuidado com a saúde psíquica de profissionais de saúde da atenção primária. Os dados foram coletados nas bases da BVS, SciELO, Lilacs e Medline, tendo como critérios de inclusão serem textos completos, em português e realizados no Brasil. Foram encontrados 7 artigos, publicados de 2010 a 2016 e desenvolvidos nas regiões Sul, Sudeste e Norte que apontam para a multifatorialidade do adoecimento, perpassando o campo pessoal e profissional. São fatores preditores para o adoecimento: a aus ência de treinamento dos profissionais, o estilo autoritário de gestão, além do fato de as características do trabalho na atenção primária provocarem elevado desgaste mental devido a grande demanda psicológica. Para a prevenção do adoecimento e dos agravos, e a promoção da saúde psíquica nos espaços de trabalho, identificou-se como dispositivos a cogestão, a educação permanente e os espaços de escuta e fala dos sofrimentos dos profissionais. Ao final, considerou -se baixa a produção bibliográfica diante de um tema de relevância para a saúde pública, reputando-se a necessidade de desenvolvimento de mais estudos na área e ampliando o escopo a fim de abranger os diferentes contextos onde estão inseridos os serviços de saúde.

\footnotetext{
1 luoliveira2005@hotmail.com

2 loyanne.chaves@gmail.com

3 magscherer@hotmail.com
} 
Palavras chave: Saúde do trabalhador; Organização do Trabalho; Saúde Mental; Atenção Primária à Saúde.

\begin{abstract}
The healthcare field is characterized by the presence of subjective conflicts that cause suffering in the professionals and can result in psychical problems, as in the case of primary care providers, which complexity of work requires dealing with situations that are often above their ability and competence. The worker's health is also influenced by the organizational model adopted in the workplace. Thus, an integrative literature review was developed with the objective of mapping caring devices with the psychic health of primary care professionals. The information was collected in BVS (Virtual Health Library), SciELO, Lilacs and Medline databases, which inclusion criteria were full texts, written in Portuguese, and having been carried out in Brazil. There we re seven articles published from 2010 to 2016 and developed in the South, Southeast and North regions, pointing to the multifactoriality of illness pervading the personal and professional areas. The lack of professional training, the authoritative management style, as well as the mental exhaustion, especially in primary care, due to the high psychological demand, are predictive factors for illness in the healthcare field. It was identified that to prevent illness and injury, it is necessary to promote mental health in the workplace through the creation of mechanisms promoting co-management, continuing professional development, and the implementation of a support system for professionals giving them the ability to share their problems and experiences. It was observed that there is a lack of texts about this subject that is so relevant to public health, and also the need to develop and expand the studies in the area including other contexts of health care.
\end{abstract}

Keywords: Worker health; Organization of Work; Mental health; Primary Health Care.

\title{
RÉSUMÉ
}

Le travail de santé se caractérise par un champ plein de conflits subjectifs, provoquant des situations de souffrance chez les professionnels, ce qui peut entraîner une maladie psychique, comme c'est le cas pour les soins primaires, où l'objet du travail est très complexe, nécessitant un traitement des situations qui échappent souvent à la compétence du professionnel. Le modèle d'organisation adopté influe également sur la maladie du travailleur. Ainsi, une revision bibliographique a été développé afin de cartographier les dispositifs de soins de santé psychique des professionnels qui travaillent dans le soins primaire. Les données ont été recueillies aux bases de la BVS, SciELO, Lilacs et Medline, les critères d'inclusion étant des textes complets, en portugais et réalisés au Brésil. Nous avons trouvé 7 articles, publiés de 2010 à 2016 et développés dans les régions du Sud, du Sud-Est et du Nord, qui indiquent la multifactorialité de la maladie, passant par le domaine personnel et professionnel. Le manque de formation des professionnels, le modèle de gestion et la posture autoritaire de la gestion, ainsi que les caractéristiques du travail dans les soins primaires qui causent un épuisement mental élevé en raison de la grande demande psychologique, sont des facteurs prédictifs pour la maladie. Pour la prévention de la maladie et pour la promotion de la santé psychique sur le lieu de travail, la gestion partagée, l'éducation permanente et la mise en place des espaces d'écoute et de sensibilisation aux problèmes des professionnels ont été identifiés comme des dispositifs. À la fin, la production bibliographique sur ce sujet a été considérée faible dans le domaine de la santé publique, compte tenu la nécessité d'élargir les études dans la région dans des différents contextes des services de santé.

Mots-clés: santé au travail; Organisation du travail; La santé mentale; Soins de santé primaires.

\section{RESUMEN}

El trabajo en salud se caracteriza por ser un campo en el que los profesionales enfrentan conflictos subjetivos, lo que ocasiona situaciones de sufrimiento que pueden resultar en enfermedades. Es el caso de la atención primaria, en la que el objeto del trabajo es de gran complejidad, exige lidiar con situaciones que a menudo desafían la capacidad y la competencia del profesional. También influye en la enfermedad del trabajador el modelo organizacional adoptado. Por lo tanto, se desarrolló una revisión integ rativa para mapear dis positivos de cuidado de la salud mental de profesionales de salud de la atención primaria. Los datos fueron recolectados en las bases de la BVS, SciELO, Lilacs y Medline; los criterios de inclusión consideran textos completos, en port ugués y realizados en Brasil. Se han encontrado 7 artículos, publicados de 2010 a 2016 y desarrollados en las regiones Sur, Sudeste y Norte, que apuntan a la multifactorialidad de la enfermedad, pasando por el campo personal y profesional. Son factores predictores para la enfermedad: la ausencia de entrenamiento de los profesionales, el estilo autoritario de la gestión, además del hecho de que las característicsas del trabajo en la atención primaria provocan desgaste mental por su gran demanda psicológica. Para la prevención de la enfermedad y la promoción 
de la salud mental en los espacios de trabajo, se identificó como dispositivos la co-gestión, la educación permanente y los espacios de escucha y habla de los sufrimientos de los profesionales. Al final, s e consideró baja la producción bibliográfica frente a un tema de relevancia para la salud pública, reputándose la necesidad de desarrollar más estudios en el área y ampliando el alcance a fin de abarcar los diferentes contextos donde están insertos los servicios de salud.

Palabras clave: Salud del trabajador; Organización del Trabajo; Salud mental; Atención Primaria a la Salud.

\section{INTRODUÇÃO}

O trabalho, entendido como uma ação de modificar a natureza para alcançar um objetivo, acompanha a história da humanidade. $\mathrm{O}$ homem, nesse processo, faz uso de instrumentos e conhecimentos que possibilitem essa transformação, e que, ao final, transforma também o próprio indivíduo (Scherer, 2006). No âmbito da saúde, a concepção de trabalho ganha uma peculiaridade que se deve ao fato de o objeto de trabalho consistir em um indivíduo, portador de necessidades de saúde (Gonçalves, 1992 apud Peduzzi \& Schraiber, 2016). Cada necessidade é singular e complexa, embora possa apresentar algumas semelhanças entre um caso e outro, demandando, portanto, atenção distinta (Scherer, 2006).

Com a especialização do trabalho ao longo da história, sobretudo na área da saúde, foram se constituindo grupos heterogêneos de trabalhadores para atender uma demanda que se complexificou com o avançar da ciência e com as transformações ocorridas nas sociedades e na natureza. Essa especialização na saúde coloca problemas para a administração do cuidado na medida em que cada profissional detém um conhecimento específico e que é relacionado com o conhecimento do outro. Nessa relação é criada uma correlação que tira autonomia de decisão do trabalhador, em diferentes proporções, podendo resultar em tensões (Carreiro, Filha, Lazarte, Silva \& Dias, 2013; Scherer, 2006).

O campo do trabalho em saúde é configurado então como um espaço repleto de conflitos que permeiam as mais diversas áreas, principalmente os serviços públicos de atenção à saúde: conflitos entre equipe, conflitos políticos, econômicos, e até sociais, e que podem, em certo grau, resultar em conflitos subjetivos (Carreiro et al., 2013; Maissiat, Lautert, Pai \& Tavares, 2015; Rosenstock, Santos \& Guerra, 2011). Os conflitos subjetivos são instaurados na medida em que as identidades construídas socialmente e impressas como normas, e que são apreendidas e absorvidas ao longo dos anos nas relações, em um determinado momento, numa vivência específica do indivíduo, afeta o seu psíquico, este entendido como a relação do sujeito com o seu sentimento (Santos, 2007).

Cristophe Dejours criou e desenvolveu, na década de 90, a teoria da Psicodinâmica do Trabalho, que se embasa na análise dos contextos de trabalho onde atuam "forças visíveis e invisíveis, objetivas e subjetivas, psíquicas, sociais, políticas e econômicas que podem ou não deteriorar esse contexto, transformando-o em lugar de saúde e/ou de patologias e de adoecimento" (Mendes, 2007, p. 29). Nesse sentido, quanto aos conflitos vivenciados pelos profissionais, entendese, à luz da teoria de Dejours, como situações de sofrimento que podem produzir o sofrimento criativo, que é a busca pela transformação das situações adversas e ressignificação do trabalho, ou as estratégias defensivas que, quando esgotadas, evidenciam o distúrbio mental, ou o adoecimento psíquico (Mendes, 2007). As situações de sofrimento são constantes na medida em que há uma grande distância entre o trabalho prescrito e o real, e nesse sentido, é válido ressaltar que a vivência do trabalho se relaciona de modo afetivo com o sujeito (Moraes, 2013). 
Autores como Braga, Carvalho e Binder (2010), Carreiro et al. (2013), e Maissiat et al. (2015) apontam que a precarização do trabalho tem feito com que haja complicações nas condições de saúde e transformação das formas de adoecer dos profissionais, aumentando as doenças relacionadas aos aspectos psicológicos, ocasionando transtornos mentais comuns (TMC), tais como ansiedade, e síndrome do esgotamento profissional (burnout), para citar alguns.

$\mathrm{O}$ adoecimento psíquico se torna uma possibilidade quando há o enrijecimento da organização do trabalho, esta entendida como a base para a realização de uma ação com um determinado fim, um esforço (Moraes, 2013). No âmbito da atenção primária à saúde, que se tornou a principal porta de entrada do usuário no Sistema Único de Saúde, o trabalho se reveste de grande complexidade devido à necessidade de ampliação do olhar profissional para a busca da integralidade do cuidado e humanização das ações, por meio da escuta qualificada, do acolhimento às necessidades dos usuários e da construção do vínculo com a comunidade local, aproximando o profissional e o usuário (Carreiro et al., 2013; Maissiat et al., 2015). As características apresentadas por esse tipo de atenção, embora desejável, trazem como possíveis consequências a sensação, por parte dos profissionais, de impotência, quando lidando com problemas que fogem da sua governabilidade em sua totalidade ou parcialmente (Maissiat et al., 2015, p. 43).

$$
\text { Mediante as diversas }
$$

responsabilidades delegadas a esses profissionais, a organização do trabalho, no âmbito da gestão do trabalho da equipe ou unidade, pode favorecer o surgimento ou agravamento do adoecimento psíquico, principalmente se o gestor responsável não estiver atento ao cuidado com os seus profissionais (Carreiro et al., 2013). Considerando o profissional como um agente transformador, é imprescindível dispor de dispositivos de cuidado com a sua saúde, não só física, mas psíquica também, objeto de estudo deste artigo.

\section{MÉTODO}

Trata-se de um estudo de revisão integrativa com vistas a ofertar uma análise dos estudos sobre o assunto desenvolvidos até então, possibilitando tomadas de decisões na área e o aprofundamento do tema através do agrupamento e sintetização dos resultados das pesquisas. Esse tipo de método visa também evidenciar lacunas de conhecimento que devem ser preenchidas em estudos futuros (Mendes, Silveira \& Galvao, 2008).

Nesse sentido, o método depreende seis passos para a sua realização, sendo eles o estabelecimento de hipótese ou questão de pesquisa; a amostragem ou busca na literatura cientifica; a categorização dos estudos; a avaliação dos estudos incluídos na revisão; a interpretação dos resultados; e a síntese do conhecimento ou apresentação da revisão (Mendes et al., 2008).

Os estudos coletados para essa revisão se encontram nas bases Biblioteca Virtual em Saúde (BVS), Scientific Electronic Library Online (SciELO), Literatura Latino-Americana e do Caribe em Ciências da Saúde (Lilacs) e Medical Literature Analysis and Retrieval System Online (Medline), utilizando-se de descritores combinados com a utilização dos operadores booleanos "AND" e "OR". Para a definição de descritores semelhantes com vistas a melhorar a busca de artigos, procurou-se no DeCS (Descritores em Ciências da Saúde) da BVS, e selecionouse os que melhor se encaixavam na proposta do estudo: ("adoecimento psíquico" $O R$ "saúde mental") AND ("recursos humanos em saúde" $O R$ "trabalhadores de saúde") $A N D$ "saúde do trabalhador" AND ("Atenção Primária à Saúde" $O R$ "Atenção Básica à Saúde") $A N D$ ("promoção da saúde" $O R$ "promoção em saúde"). 
Foram encontrados um total de 21 artigos que abordavam o adoecimento ou saúde psíquica dos profissionais de saúde da APS, explícitos no título ou nos resumos dos estudos. Os critérios de seleção de artigos foram: textos completos disponíveis; escritos em português; e Brasil como campo de estudo. Mediante os achados, por serem poucos os estudos encontrados, considerou-se incluir todos os artigos levantados, sem delimitação de tempo e cenário. Ao total, incluiu-se então os 7 artigos publicados que, retirando-se os duplicados e presentes em mais de uma base de dados, foram selecionados pelo título e resumo mediante a abordagem do objeto de estudo.

De posse dos artigos, as informações extraídas foram organizadas em uma matriz analítica que continha os seguintes eixos: ano do estudo; participantes da pesquisa; objetivos; metodologia; resultados da pesquisa desenvolvida; conclusões dos pesquisadores; dispositivos de prevenção das causas do adoecimento; e dispositivos promotores da saúde no ambiente de trabalho.

\section{RESULTADOS}

Os artigos encontrados são dos anos de 2010 a 2016, publicados um em cada ano em revistas de São Paulo (SP), Rio de Janeiro (RJ) e Rio Grande do Sul (RS), com estudos desenvolvidos em SP, RS, Minas Gerais (MG), Santa Catarina (SC) e Amazonas (AM). Embora tenha a presença do estado do AM, o estudo desenvolvido por esses autores, em conjunto com SC, foi de cunho teórico. Com base na revisão bibliográfica, quatro dos sete artigos encontrados tiveram como objetivo a identificação da relação entre condições de trabalho e situações de adoecimento psíquico, entre os quais foram citados a Síndrome de Burnout, Transtornos Mentais Comuns (TMC) e transtornos psiquiátricos menores. Para isso, foi desenvolvido um estudo transversal descritivo com análise estatística aplicando questionário ou realizando entrevista com profissionais da APS para a obtenção dos dados (Alcântara \& Assunção, 2016; Braga et al., 2010; Dilélio et al., 2012; Silveira, Câmara \& Amazarray, 2014).

Apenas um dos artigos cruzou a avaliação do contexto do trabalho com os indicadores de prazer e sofrimento na perspectiva dos profissionais, indicadores esses que integram a discussão abordada por Dejours em sua teoria voltada para o tema. Nesse sentido, os autores desenvolveram um estudo transversal com análise estatística descritiva e inferencial, coletando dados por meio de um questionário (Maissiat et al., 2015). Em outros dois estudos identificados na revisão, foi realizado ensaio teórico. Um deles tinha como finalidade discutir possibilidades de intersetorialidade entre saúde mental e saúde do trabalhador na atenção básica (Bernardo \& Garbin, 2011). $\mathrm{O}$ segundo estudo buscou fazer uma análise à luz da Teoria da Psicodinâmica do Trabalho de Dejours sobre o sofrimento psíquico do trabalhador da Estratégia de Saúde da Familia (ESF) - que faz parte do plano de reorientação da atenção primária (Katsurayama et al., 2013).

Bernardo e Garbin (2011), apesar de não se restringirem a atenção à saúde mental decorrente do trabalho de profissionais de saúde, trazem à tona a cultura da discriminação ao adoecimento mental no trabalho, inclusive entre profissionais que prestam o atendimento aos enfermos. Isso se deve a "cultura da positividade do trabalho no contexto capitalista e a naturalização dos acidentes e doenças decorrentes do trabalho" (p.110), além de que, para a população leiga, apenas os acidentes visíveis tornam passíveis a sua sensibilização. Além disso, os autores apontam para o pequeno escopo de atuação da atenção à saúde mental, que focou em desconstruir a cultura manicomial. Consideram de grande importância essa luta, mas a ampliação da 
atenção é imprescindível quando é constatado, por meio da leitura dos estudos desenvolvidos até então, a atual condição do estado de saúde mental dos trabalhadores. Diante das apurações, os autores concluem que há dificuldades de integrar essas duas grandes áreas pela divergência de eixo de atuação, e principalmente pela questão cultural envolvida (Bernardo \& Garbin, 2011).

Os resultados dos estudos analíticos identificados apontam que o motivo para o adoecimento psíquico está relacionado ao elevado desgaste mental devido à alta demanda psicológica que envolve o trabalho, pressupondo que essa condição se deva pelas próprias características do processo de trabalho e pelas situações as quais os profissionais se deparam, como as agressões, por exemplo. A isso, soma-se a ausência de treinamento e o modelo de gestão em vigor, que não busca fazer convergir os interesses, as expectativas e os recursos dos atores envolvidos (profissionais e gestão) no planejamento conjunto do trabalho segundo as realidades vivenciadas (Alcântara \& Assunção, 2016; Braga et al., 2010; Dilélio et al., 2012; Silveira et al., 2014).

Katsurayama et al. (2013) afirmam que as patologias referentes à sobrecarga no trabalho têm se elevado na atualidade, tendo o sistema hierárquico e a divisão de tarefas como importantes fontes de sofrimento. Diante do exposto, os autores afirmam que o espaço oferecido pela gestão para a discussão da organização entre os profissionais é imprescindível para a mobilização subjetiva e transformação das condições patogênicas, principalmente na atenção básica, onde há elevada demanda psicológica (Katsurayama et al., 2013).

Fatores associados à vida pessoal do profissional também foram identificados como elementos que predispõem o adoecimento psíquico, como por exemplo, as questões familiares e até mesmo a ausência da prática de atividades físicas (Dilélio et al, 2012; Silveira et al.,
2014). No entanto, eles tornam possível o adoecimento quando associados a outros fatores, apontando para a multifatorialidade dessa condição (Silveira et al., 2014).

Entendendo dispositivo ${ }^{4}$ como àquilo que propicia algum acontecimento ou ocorrência desejada, ou seja, algo que faça advir um efeito esperado (Campos, 2003 apud Jesus \& Assis, 2010), os autores indicaram para o provimento de recursos (tanto materiais quanto organizacionais), os mecanismos de formação continuada e espaços de discussão como dispositivos preventivos do adoecimento psíquico na medida em que constituem importantes fontes de prazer, principalmente quando esses espaços de discussão permitem a fala e a escuta dos sujeitos e a transformação das fontes de sofrimento, que podem significar a transformação do trabalho prescrito e até mesmo da organização em vigor (Alcântara \& Assunção, 2016; Braga et al, 2010; Dilélio et al., 2012; Katsurayama et al., 2013; Maissiat et al, 2015; Silveira et al., 2014). Além dos já citados, o acolhimento ao trabalhador - e que pressupõe o reconhecimento, que deve ser tanto pela "hierarquia" quanto pelos pares - e a construção de um projeto comum também foram identificados como dispositivos de prevenção do adoecimento (Katsurayama et al., 2013; Maissiat et al, 2015).

No tocante à promoção da saúde psíquica dos profissionais, os autores indicam o incentivo à confiança e solidariedade entre pares, seguido pelo reconhecimento do trabalho que pressupõe a sua valorização (Dilélio et al., 2012; Katsurayama et al., 2013).

Entendendo o adoecimento psíquico como uma condição multifatorial, Dilélio et al. (2012) e Silveira et al. (2014) asseguram a importância de $\mathrm{o}$ gestor valorizar $o$ equilibrio temporal entre $o$ trabalho e a vida familiar e social dos

4 Neste artigo utilizamos dispositivos como sinônimos de modos, meios, formas para se atingir um fim. 
trabalhadores, haja vista que são importantes fatores para a manutenção da saúde mental do profissional.

\section{DISCUSSÃO}

Constatou-se, por meio da revisão bibliográfica, que o olhar para o sofrimento psíquico de trabalhadores da saúde da Atenção Primária tem se mostrado tímido no meio científico, sobretudo nas regiões Norte e Centro-Oeste, onde não se identificou estudos de cunho experimental desenvolvidos.

Profissionais de saúde da atenção primária atuam em um campo de cuidado complexo que demanda muito do psicológico desses sujeitos, que propicia desgaste mental com sobrecarga emocional devido a relação do processo de trabalho com determinantes e condicionantes da saúde do usuário, que muitas vezes ultrapassam o campo de competências do profissional e, consequentemente, a resolubilidade do problema, resultando em sofrimentos que podem levar ao adoecimento psíquico (Rosenstock, 2011). Aliado a essa sobrecarga mental existem os elementos da vida pessoal do indivíduo que influenciam, em maior ou menor grau, na condição de adoecer ou não, e que não podem ser desconsiderados quando se busca dispositivos que protejam e promovam a saúde psíquica desses profissionais (Dilélio et al., 2012; Silveira et al., 2014).

Em geral, o profissional por si só busca e/ou desenvolve mecanismos que o auxiliem no combate ao sofrimento, seja enfrentando-o como problema, por intermédio do sofrimento criativo, ou negando-o enquanto problema, criando estratégias defensivas, e que estas, por sua vez, podem resultar no adoecimento (Mendes, 2007).

Considerando a multifatorialidade que envolve esse tipo de adoecimento, a postura da gestão e o modelo organizacional adotados podem auxiliar $\mathrm{o}$ trabalhador na superação do sofrimento por intermédio da transformação dos espaços e relações causadores de sofrimento ou, ao contrário, ignorando os fatores que influenciam nesse adoecimento, instar-se neutro nesse processo ou até mesmo contribuir para o adoecimento aumentando a sobrecarga de trabalho, que podem ter como consequência as licenças médicas decorrentes e os desengajamentos resultantes do processo do adoecer, resultando em um serviço com menor capacidade de atenção e resolubilidade. Diante do exposto, considera-se que a flexibilidade do gestor para a discussão e revisão dos processos de trabalho para readequação da organização com a realidade, atento às necessidades dos usuários e podem favorecer os sentimentos de prazer dos trabalhadores, que por sua vez poderão influenciar na qualidade do atendimento e resolubilidade das demandas de saúde (Katsurayama et al., 2013). Depreende-se então a necessidade de se equalizar as demandas da gestão com os desejos dos usuários e dos profissionais, proposta essa apresentada pela Política Nacional de Humanização (PNH), resultante da compreensão da presença de outras dimensões que influem nas práticas de atenção, como as dimensões sociais e subjetivas (Ministério da Saúde, 2004).

Nesse sentido, a cogestão conceituada por Guizardi e Cavalcanti (2010, apud Campos, 2000) visa o compartilhamento de poder com vistas à democratização das decisões envolvidas na organização do trabalho e na busca pela superação dos dispositivos e estratégias de dominação e controle que não permitem diminuir a distância entre o trabalho prescrito e o real. Depreende-se que a cogestão pode atuar como um importante dispositivo de resignificação do trabalho, e consequentemente promotor da saúde psíquica, na medida em que permite os espaços de fala e escuta, importante fator destacado como propulsor para o adoecimento.

Nos espaços de cogestão é válido considerar as perspectivas sobre os 
processos de trabalho e a sua finalidade a fim de evidenciar um objetivo consensual entre a equipe, e a partir daí construir um planejamento com vistas a orientar a ação dos trabalhadores concomitante com a avaliação dos resultados dos objetivos propostos, visando o acompanhamento da dinamicidade presente nos processos de trabalho em saúde (Correia, 2011).

Ainda sobre esses espaços, na medida em que ele traz à luz a realidade do trabalho, em como os trabalhadores produzem as ações de saúde e os seus respectivos resultados, podem dar lugar também a um desconforto no profissional, que possui expectativas, metas, objetivos. Nesse sentido, problematizar tais realidades em relação aos princípios norteadores do Sistema Único de Saúde (SUS) potencializa a transformação e o desenvolvimento de ações que qualificam a atenção, e pode contribuir para aumentar o reconhecimento e a valorização do trabalho e, principalmente, do trabalhador (Ceccim \& Ferla, 2016). Além disso, Katsurayama et al. (2013) afirmam que analisar o sofrimento psíquico dos profissionais pode auxiliar na identificação de entraves e indicação de caminhos de superação na busca por uma atenção básica integral e de qualidade.

Por fim, Ceccim e Ferla (2016), ao evidenciarem a potencialidade da educação permanente em saúde, convergem com o encontrado na revisão de literatura quando identificam tal ação como um dispositivo necessário para a proteção e a promoção da saúde psíquica dos profissionais, haja vista que se trata de uma "prática de ensinoaprendizagem" na medida em que, a partir das experiências dos trabalhadores, produz-se conhecimento na finalidade de qualificar tais práticas.

\section{SÍNTESE DO CONHECIMENTO}

Os estudos apontaram para uma condição multifatorial para o adoecimento psíquico, onde a postura do gestor e a organização do trabalho tem se mostrado como importantes propulsores para o agravamento ou redução desse adoecimento. Nesse sentido, a busca pela transformação do trabalho com vistas a aproximação do que é prescrito com o real é de extrema importância para a possibilidade de sustentabilidade da atenção à saúde.

Algumas posturas e ações, e que podem ser consideradas como dispositivos, foram apontadas pelos autores como promotoras da saúde psíquica desses profissionais, dentre os quais o conceito de cogestão aliado ao planejamento das ações, e a educação permanente em saúde, pressupondo, por fim, a importância da postura adotada pelo gestor da(s) equipe(s) de saúde para a qualidade da atenção e do ambiente de trabalho, para que seja saudável psiquicamente.

Considerou-se baixa a produção científica diante da relevância da temática para a saúde pública, mas se compreende que é um campo recém-descoberto, com o desenvolvimento de teorias voltadas para o assunto há pouco mais de duas décadas. Portanto, reputa-se como imprescindível o desenvolvimento de mais estudos no âmbito da saúde psíquica de profissionais de saúde da APS que busquem a relação com a teoria já desenvolvida, e buscando a integração das áreas de Saúde do Trabalhador e Saúde Mental.

Para o desenvolvimento de novos estudos se julga necessário a ampliação do escopo para abranger as demais regiões não estudadas, com vistas a analisar tais argumentos em outros contextos, buscando o aumento da qualidade do serviço em diferentes realidades e analisando-o longitudinalmente para uma apreciação mais próxima dos processos de sofrimento no trabalho e avaliação da efetividade das intervenções.

\section{Referências}


Alcântara, M.A. \& Assunção, A.A. (2016). Influência da organização do trabalho sobre a prevalência de transtornos mentais comuns dos agentes comunitários de saúde de Belo Horizonte. Rev Bras Saúde Ocup, v. 41, e.2, Retrieved in August 22, 2016, from http://www.scielo.br/scielo.php?pid= $\underline{\text { S030376572016000100202\&script }=S}$ ci abstract\&tlng=pt.

Bernardo, M.H. \& Garbin, A.D.C. (2011). $\mathrm{A}$ atenção à saúde mental relacionada ao trabalho no SUS: desafios e possibilidades. Rev Bras Saúde Ocup, v. 36, n. 123, Retrieved in June, 2011, from http://www.scielo.br/scielo.php?scrip t=sci_arttext\&pid=S0303$\underline{76572011000100010 .}$.

Braga, L.C., Carvalho, L.R. \& Binder, M.C.P. (2010). Condições de trabalho e transtornos mentais comuns em trabalhadores da rede básica de saúde de Botucatu (SP). Ciência \& Saúde Coletiva, v. 15, supl. 1, Retrieved in June, 2010, from

http://www.scielo.br/scielo.php?scrip $\mathrm{t}=$ sci_arttext\&pid=S1413$\underline{81232010000700070 .}$.

Brasil. Ministério da Saúde. Núcleo Técnico da Política Nacional de Humanização. (2004). HumanizaSUS: Política Nacional de Humanização: a humanização como eixo norteador das práticas de atenção e gestão em todas as instâncias do SUS. Brasilia: Ministério da Saúde.

Carreiro, G.S.P., Filha, M.O.F., Lazarte, R., Silva, A.O. \& Dias, M.D. (2013). $\mathrm{O}$ processo de adoecimento mental do trabalhador da Estratégia Saúde da Familia. Rev Eletr Enf, v. 15, n. 1, Retrieved in March, 2013, from https://www.fen.ufg.br/fen_revista/v 15/n1/pdf/v15n1a17.pdf.

Correia, A.D.M.S. et al. (2011). Integralidade na atenção à saúde. In: Pós-Graduação em Atenção Básica em Saúde da Família. UFMS.

Dilélio, A.S., Facchini, L.A., Tomasi, E., Silva, S.M., Thumé, E., Piccini, R.X., Silveira, D.S., Maia, M.F.S., Osório, A., Siqueira, F.V., Jardim, V.M.R., Lemões, M.A.M. \& Borges, C.L.S. (2012). Prevalência de transtornos psiquiátricos menores em trabalhadores da atenção primária à saúde das regiões Sul e Nordeste do Brasil. Cad Saúde Pública, v. 28, n. 3, Retrieved in March, 2012, from http://www.scielo.br/scielo.php?scrip $\mathrm{t}=\mathrm{sci}$ arttext\&pid=S0102311X2012000300011.

Fundação Oswaldo Cruz \& Escola Politécnica de Saúde Joaquim Venâncio (2016). Educação permanente em saúde. In: Dicionário da Educação Profissional em Saúde. CECCIM, R.B. \& FERLA, A.A. From

http://www.epsjv.fiocruz.br/dicionari o/index.html.

Fundação Oswaldo Cruz \& Escola Politécnica de Saúde Joaquim Venâncio (2016). Processo de Trabalho em Saúde. In: Dicionário da Educação Profissional em Saúde. PEDUZZI, M. \& SCHRAIBER, L.B. From

http://www.epsjv.fiocruz.br/dicionari o/index.html.

Guizardi, F.L. \& Cavalcanti, F.O.L. (2010). O conceito de cogestão em saúde: reflexões sobre a produção de democracia institucional. Physis Rev de Saúde Coletiva, v. 20, n. 4, Retrivied in December, 2010, from http://www.scielo.br/scielo.php?scrip 
$\mathrm{t}=$ sci_arttext\&pid=S010373312010000400010.

Jesus, W.L.A. \& Assis, M.M.A. (2010). Revisão sistemática sobre o conceito de acesso nos serviços de saúde: contribuições do planejamento. Rev Ciência \& Saúde Coletiva, v. 14, n. 1, Retrivied in January, 2010, from http://www.scielo.br/scielo.php?scrip $\mathrm{t}=$ sci arttext\&pid=S141381232010000100022.

Katsurayama, M., Parente, R.C.P., Moraes, R.G. \& Moretti-Pires, R.O. (2013). Trabalho e sofrimento psíquico na Estratégia Saúde da Familia: uma perspectiva Dejouriana. Cad. Saúde Colet., v. 21, n. 4, Retrivied in 2013, from

http://www.scielo.br/scielo.php?scrip $\mathrm{t}=$ sci_arttext\&pid=S1414462X2013000400009.

Maissiat, G.S., Lautert, L., Pai D.D. \& Tavares, J.P. (2015). Contexto de trabalho, prazer e sofrimento na atenção básica em saúde. Rev Gaúcha Enferm, v. 36, n. 2, Retrivied in June, 2015, from http://www.scielo.br/scielo.php?pid= S1983-14472015000200042\& script=sci_arttext\&tlng=pt.

Mendes, A.M. (2007). Da psicodinâmica à psicopatologia do trabalho. In: MENDES, A.M. (Org.). Psicodinâmica do Trabalho: teoria, método e pesquisas. São Paulo: Casa do Psicólogo. p. 29.

Mendes, K.D.S., Silveira, R.C.C.P. \& Galvao, C.M. (2008). Revisão integrativa: método de pesquisa para a incorporação de evidências na saúde e na enfermagem. Rev Texto contexto - enferm, v. 17, n. 4, Retrivied in December, 2008, from http://www.scielo.br/scielo.php?scrip
t=sci_arttext\&pid=S0104$\underline{07072008000400018 .}$.

Moraes, R.D. (2013). Sofrimento Criativo e Patogênico. In F. O. Vieira, A. M. Mendes, \& A.R.C. Merlo (Orgs.), Dicionário Crítico de Gestão e Psicodinâmica do Trabalho (pp. 415-420). Curitiba: Juruá.

Rosenstock, K.I.V.; Santos, S.R. \& Guerra, C.S. (2011). Motivação e envolvimento com o trabalho na Estratégia Saúde da Familia em João Pessoa, Paraíba, Brasil. Rev Baiana de Saúde Pública, v. 35, n. 3, Retrivied in September, 2011, from http://files.bvs.br/upload/S/01000233/2011/v35n3/a2638.pdf.

Santos. L.O. (2007). "Transtorno de Pânico", Um Estudo sobre as Matrizes Sociais de seu Surgimento: a sociedade do risco e a construção contemporânea de bioidentidades. 197 f. Tese (Doutorado em Saúde Coletiva) - Instituto de Medicina Social, Universidade do Estado do Rio de Janeiro, Rio de Janeiro.

Scherer, M.D.A (2006). O trabalho da equipe no Programa de Saúde da Família: possibilidades de construção da interdisciplinaridade. 232 f. Tese (Doutorado em Enfermagem) - Centro de Ciências da Saúde, Universidade Federal de Santa Catarina, Florianópolis.

Silveira, S.L.M., Câmara, S.G. \& Amazarray, M.R. (2014). Preditores da Síndrome de Burnout em profissionais da saúde na atenção básica de Porto Alegre/RS. Cad. Saúde Colet., v. 22, n. 4, Retrivied in December, 2014, from http://www.scielo.br/scielo.php?pid= S1414$\underline{462 X 2014000400386 \& \text { script }=\text { sci_ab }}$ stract\&tlng=pt. 
Moraes, R.D. (2013). Sofrimento Criativo e Patogênico. In F. O. Vieira, A. M. Mendes, \& A.R.C. Merlo (Orgs.), Dicionário Crítico de Gestão e Psicodinâmica do Trabalho (pp. 415-420). Curitiba: Juruá.

Data de submissão: 11/10/2017

Data de aceite: $\quad$ 01/11/2017 